\title{
2.5-Dimensional finite-difference time-domain analysis for propagation of conventional railway noise: Application to propagation of sound from surface railway and its verification by scale model experiments
}

\author{
Masaaki Hiroe ${ }^{1, *}$, Tomohiro Kobayashi ${ }^{1}$ and Satoshi Ishikawa ${ }^{2}$ \\ ${ }^{1}$ Kobayasi Institute of Physical Research, \\ 3-20-41 Higashi-motomachi, Kokubunji, 185-0022 Japan \\ ${ }^{2} J R$ East Consultants Co./Graduate School of Frontier Sciences, University of Tokyo, \\ JR Shinjyuku Bldg. 6F, 2-2-6 Yoyogi, Shibuya-ku, Tokyo, 151-0053 Japan
}

(Received 5 July 2016, Accepted for publication 16 September 2016)

Keywords: 2.5D-FDTD analysis, Conventional railway noise, Sound propagation, Noise barrier, Shielding effect PACS number: 43.50.Gf, 43.28.Js [doi:10.1250/ast.38.42]

\section{Introduction}

Noise sources at the lower parts of trains, such as bogie noise, rolling noise, and fan noise of equipment, are one of the most important sources of train noise for a conventional railway. Various barriers were developed to reduce noise from the lower parts of trains. In our previous study [1], we estimated the shielding effect of barriers against the noise radiated from the lower parts of a train by the two-dimensional finite-difference time-domain (2D-FDTD) method. Although the source of such noise is generally considered to be an incoherent line source, we usually treated such noise sources as a coherent line source in order to simplify the complex acoustic problem and reduce the calculation cost. On the other hand, 2.5-dimensional (2.5D) numerical analysis has recently been applied to a road traffic noise problem in which the noise is radiated from a single car with a uniform crosssectional view along the length of a road. This numerical technique is a method of transforming a two-dimensional solution to a three-dimensional one based on Duhamel's transformation method using the solution obtained by the boundary element method (2.5D-BEM) [2] or the FDTD method (2.5D-FDTD) [3].

In this report, we applied 2.5D-FDTD analysis to calculate the propagation of noise radiated from the lower parts of the train for a conventional surface railway. Moreover, in order to verify the accuracy of numerical results obtained by the 2.5D-FDTD analysis, we carried out an experiment using a $1 / 25$ scale model of a surface railway.

\section{Numerical and experimental methods}

A distribution of sound sources, a slab track, a train body, and a noise barrier are used in this study, as shown in Fig. 1. Both numerical analysis by the FDTD method and a scale model experiment were carried out with a flat reflective ground, and the noise sources were treated as an incoherent line source with a length equivalent to the train length in this study. The sound source was assumed to have the characteristics of an A-weighted sound power spectrum, which was measured in the vicinity of a passing train. The overall Aweighted sound pressure level (SPL) was calculated at frequencies between $100 \mathrm{~Hz}$ and $2.5 \mathrm{kHz}$. The cross sections of three reflective noise barriers [Case (A): straight type; Case (B): top-bent type; Case (C): inversed-L type] are shown in Fig. 1.

2.1. 2.5D analysis by FDTD method

As shown in Fig. 2, the cross section of the railway structure with the barrier was modeled in a 2D sound field.

The sound source was set under the train body at the center of the nearest track $\left(x_{\mathrm{s}}, y_{\mathrm{s}}\right)$ and at the height of the railhead, and the initial sound pressure distribution with a Gaussian profile was used. Around the calculation area, a perfectly matched layer of $5 \mathrm{~m}$ width was set to produce a hemi-free sound field. In this study, we used a fourth-order spatial finite difference scheme [4] to reduce dispersion error in the 2D-FDTD simulation. The discrete spatial interval was set to $0.008 \mathrm{~m}$ and the time step to around $1 / 128,000 \mathrm{~s}$. We obtained the transient solution $p_{2 \mathrm{D}}(x, y, t)$ in the calculation area.

By including both the Fourier transform results $\Phi_{2 \mathrm{D}}(x, y, k)$ and Laplace transform results $\Phi_{2 \mathrm{D}}(x, y, j k)$ of the integrated functions $\varphi_{2 \mathrm{D}}(x, y, t)$ of $p_{2 \mathrm{D}}(x, y, t)$ to Duhamel's transformation, as shown in Eq. (1), we obtained the solution $\Phi_{2.5 \mathrm{D}}(x, y, z, k)$ for a point source at the receiving position $(x, y, z)$ in Cartesian coordinates with $k$ as the wave number $(=\omega / c, \omega$ is the angular frequency and $c$ is the speed of sound) [5]. $\alpha$ is a variable of integration to transform the wave number in the 2D sound field, $k^{\prime}=\sqrt{ }\left(k^{2}-\alpha^{2}\right)$, into that in a $3 \mathrm{D}$ sound field, $k$. Using the reciprocity theorem, we calculated a set of solutions at source positions $\left(x_{\mathrm{s}}, y_{\mathrm{s}}, z_{\mathrm{s}}\right)$ for $z_{\mathrm{s}}=0$ to $60 \mathrm{~m}$ at $1 \mathrm{~m}$ intervals at each point in the calculation area. By summing the squared values of sound pressure at the positions $\left(x_{\mathrm{s}}, y_{\mathrm{s}}, z_{\mathrm{s}}\right)$ corresponding to the incoherent line source, we obtained 2.5D results for the noise sources positioned under the train body.

*e-mail: hiroe@kobayasi-riken.or.jp 


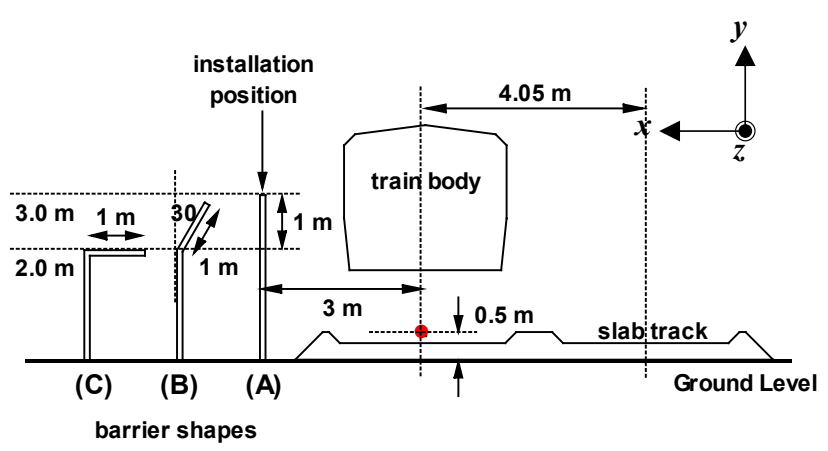

Fig. 1 Geometrical setting of a train, a noise barrier, and a surface track of railway and differently shaped noise barriers $(\mathrm{A})$ to $(\mathrm{C})$.

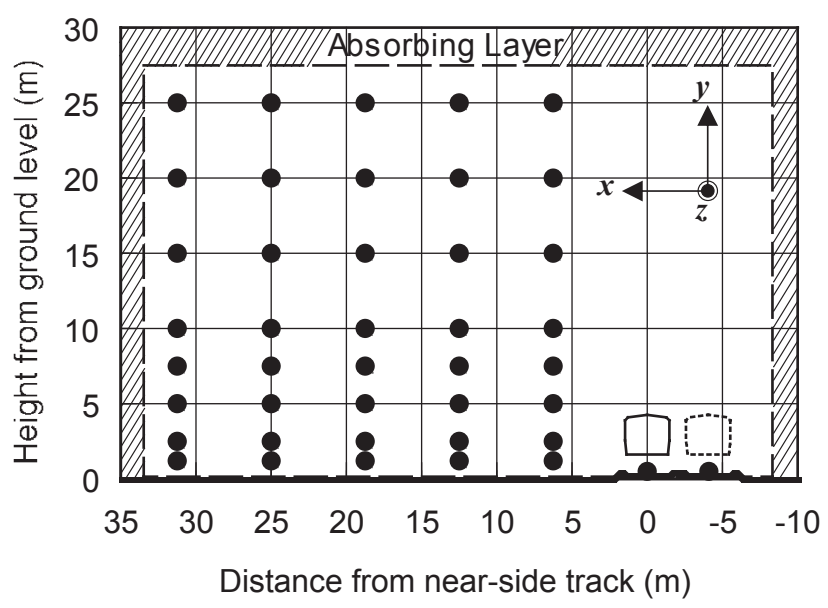

Fig. 2 Arrangement of a source, receivers, a train, and a surface track of railway for both numerical analysis by FDTD method and scale model experiments.

$$
\begin{aligned}
\Phi_{2.5 \mathrm{D}}(x, y, z, k)= & \frac{1}{\pi} \int_{0}^{k} \Phi_{2 \mathrm{D}}\left(x, y, \sqrt{k^{2}-\alpha^{2}}\right) \cos (\alpha z) d \alpha \\
& +\frac{1}{\pi} \int_{k}^{\infty} \Phi_{2 \mathrm{D}}\left(x, y, j \sqrt{\alpha^{2}-k^{2}}\right) \cos (\alpha z) d \alpha(1)
\end{aligned}
$$

\subsection{Method of experiment using scale models}

The $1 / 25$ scale models, which consisted of a train, a slab track structure, and a noise barrier, were constructed in a hemi-anechoic room. All scale models were made with acrylic boards to perfectly reflect the sound off the surface. The model represented a 120 -m-long train on the nearest track, and a 160-m-long slab track and noise barriers. A 120-m-long incoherent line source was positioned at the center of the nearest track, and the barrier was set at a distance of $3 \mathrm{~m}$ from the center towards the left side, as shown in Fig. 2. The arrangement of the measuring points in the scale model experiments is shown in Fig. 2. We kept both the temperature and humidity in the room constant, and measured 1/3-octave band levels averaged over $10 \mathrm{~s}$ in an actual frequency range from $100 \mathrm{~Hz}$ to $2.5 \mathrm{kHz}$ at each measuring point.
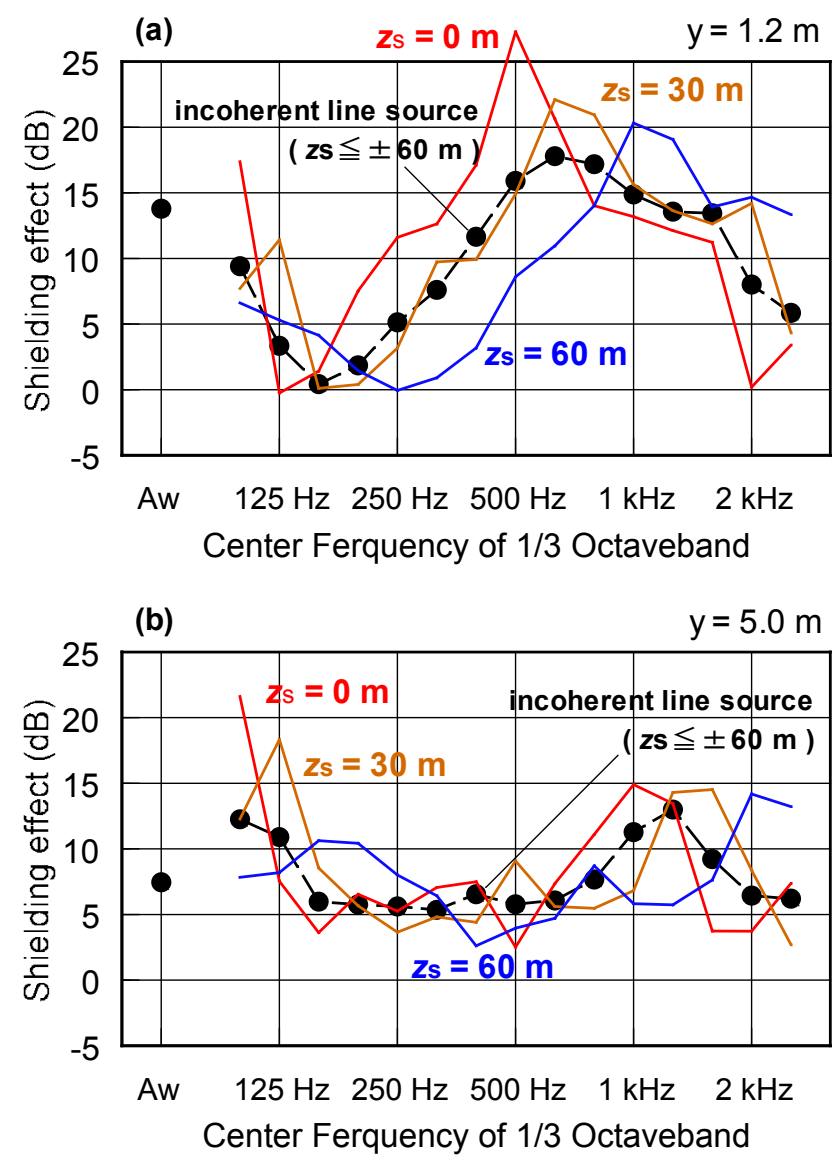

Fig. 3 Frequency characteristics of shielding effects of incoherent line source and each point source for barrier (A) at two receiving positions: (a) $x=25 \mathrm{~m}, y=1.2 \mathrm{~m}$ and (b) $x=25 \mathrm{~m}, y=5.0 \mathrm{~m}$.

\section{Comparison between numerical and experimental results and discussion}

Figure 3(a) shows the shielding effects of barrier (A) at $x=25 \mathrm{~m}$ and $y=1.2 \mathrm{~m}$ for point sources at three positions $z_{\mathrm{s}}=0,30$, and $60 \mathrm{~m}$, and Fig. 3(b) shows those at $x=25 \mathrm{~m}$ and $y=5.0 \mathrm{~m}$ for the same positions $z_{\mathrm{s}}$. From the results, we can see that the shielding effects for each point source had peak frequencies corresponding to source and receiver positions. These peak frequencies are dependent on the constructive/destructive interference of sound waves propagating between direct and ground-reflective paths. Therefore, each peak frequency shifts to a higher frequency range with increasing distance of the source position $z_{\mathrm{s}}$, because the difference between direct and ground-reflective paths decreases inversely proportionally to $z_{\mathrm{s}}$. On the other hand, the frequency characteristics of an incoherent line source were more smooth than those of a point source. There is a similar difference between insertion losses in the $2 \mathrm{D}$ and $2.5 \mathrm{D}$ numerical results [6]. These findings indicate that the calculated results for a point source or a coherent line source should be applied carefully in the prediction of conventional railway noise in a $2.5 \mathrm{D}$ sound field.

Figure 4 shows the frequency characteristics of the shielding effects for an incoherent line source obtained by 
(A)

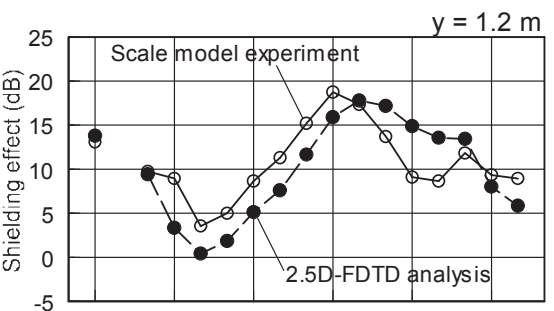

(B)

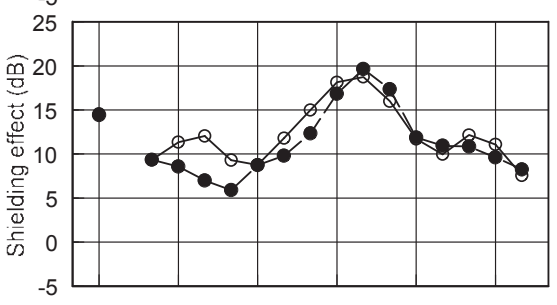

(C)

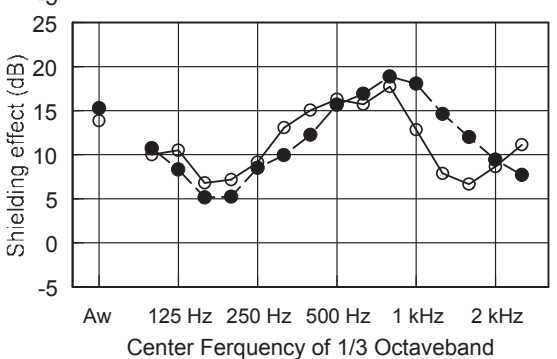

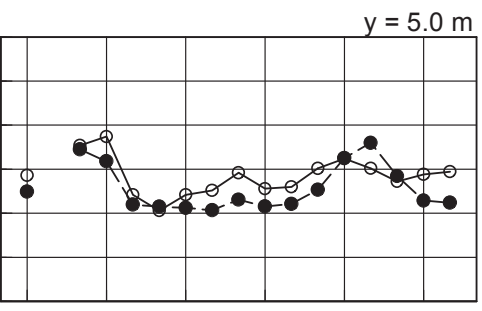
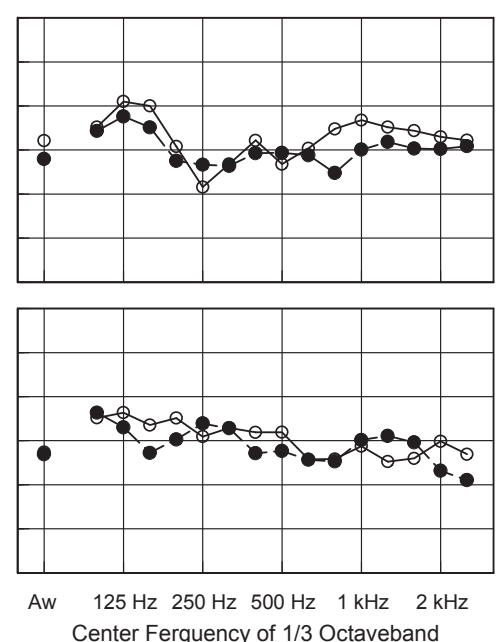
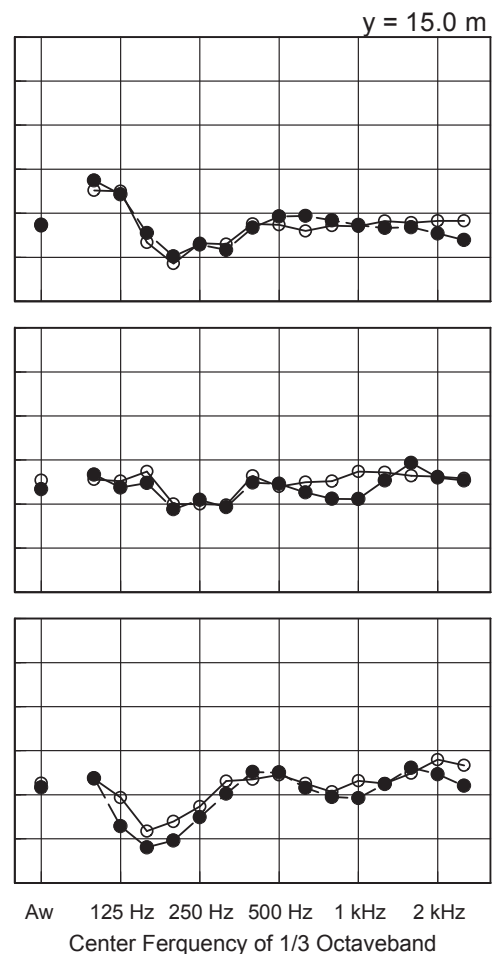

Fig. 4 Frequency characteristics of the shielding effects obtained by 2.5D-FDTD analysis and scale model experiments for barriers (A) to (C) at $x=25 \mathrm{~m}$.

the 2.5D-FDTD analysis and the scale model experiments at a distance of $x=25 \mathrm{~m}$ and heights of $y=1.2,5.0$, and $15.0 \mathrm{~m}$. From this figure, we can see that the frequency characteristics of the shielding effect smoothly change, and there is a peak frequency that depends on the height of the receiving position, that is, $500-800 \mathrm{~Hz}$ for $y=1.2 \mathrm{~m}, 100-125 \mathrm{~Hz}$ for $y=5.0 \mathrm{~m}$, and $63-80 \mathrm{~Hz}$ for $y=15.0 \mathrm{~m}$. For these frequency characteristics of the shielding effects, the 2.5D-FDTD analysis results agreed well with the experimental ones.

The overall shielding effect resulted from the differences between the overall A-weighted SPLs without and with a barrier. Figure 5 shows spatial distributions of the overall shielding effect from the 2.5D-FDTD analysis and experiment for the noise barrier conditions of Cases (A) to (C). The 2.5DFDTD analysis results also showed good agreement with those of the scale model experiment in the spatial distribution of the overall shielding effect for the conventional railway noise.

These good agreements for both frequency characteristics and spatial distribution indicate that the 2.5D-FDTD analysis is a useful tool for precisely predicting the sounds that propagate from incoherent line sources, such as noise sources of a conventional railway.

\section{Conclusions}

In order to calculate the propagation of sound emitted from a conventional railway behind a reflective barrier, we applied a numerical technique of transforming a solution for a coherent line source to a solution for a point source. This technique is based on Duhamel's method in which a transient solution obtained by the 2D-FDTD method is used, and is called the 2.5D-FDTD analysis. Moreover, we confirmed the 2.5D-FDTD analysis results through comparison with the results of an experiment using a 1/25 scale model. By comparing the shielding effects between the numerical and experimental results, the 2.5D-FDTD analysis results were found to agree with the results of the scale model experiment very well for both spatial distribution and frequency characteristics. This good agreement indicates that the 2.5D-FDTD analysis is a useful tool for precisely predicting the sounds that propagate from incoherent line sources, such as conventional railway noise. It might be caused by the summation of the squared values of sound pressures propagating from each noise source position. We should investigate the mechanism behind this problem in the future. Moreover, although the numerical analysis in this paper is restricted to the FDTD method, we should apply 2.5D-BEM [7] to the calculation of the propagation of conventional railway noise behind absorptive barriers and/or above ballast tracks in the future.

\section{References}

[1] M. Hiroe, J. Kaku, K. Mori, Y. Takakuwa and S. Nozawa, "Numerical analysis on the shielding effects of noise barriers against bogie noise by finite-difference time-domain method," Proc. Inter-Noise 2007, in07_326, Istanbul (2007).

[2] T. Okubo and K. Yamamoto, "Equivalence in diffractionreducing efficiency between T-profile and thick barrier against road traffic noise," Acoust. Sci. \& Tech., 34, 277-283 (2013).

[3] S. Sakamoto, "Development of energy-based calculation methods of noise radiation from semi-underground road using a numerical analysis," Acoust. Sci. \& Tech., 31, 75-86 (2010). 

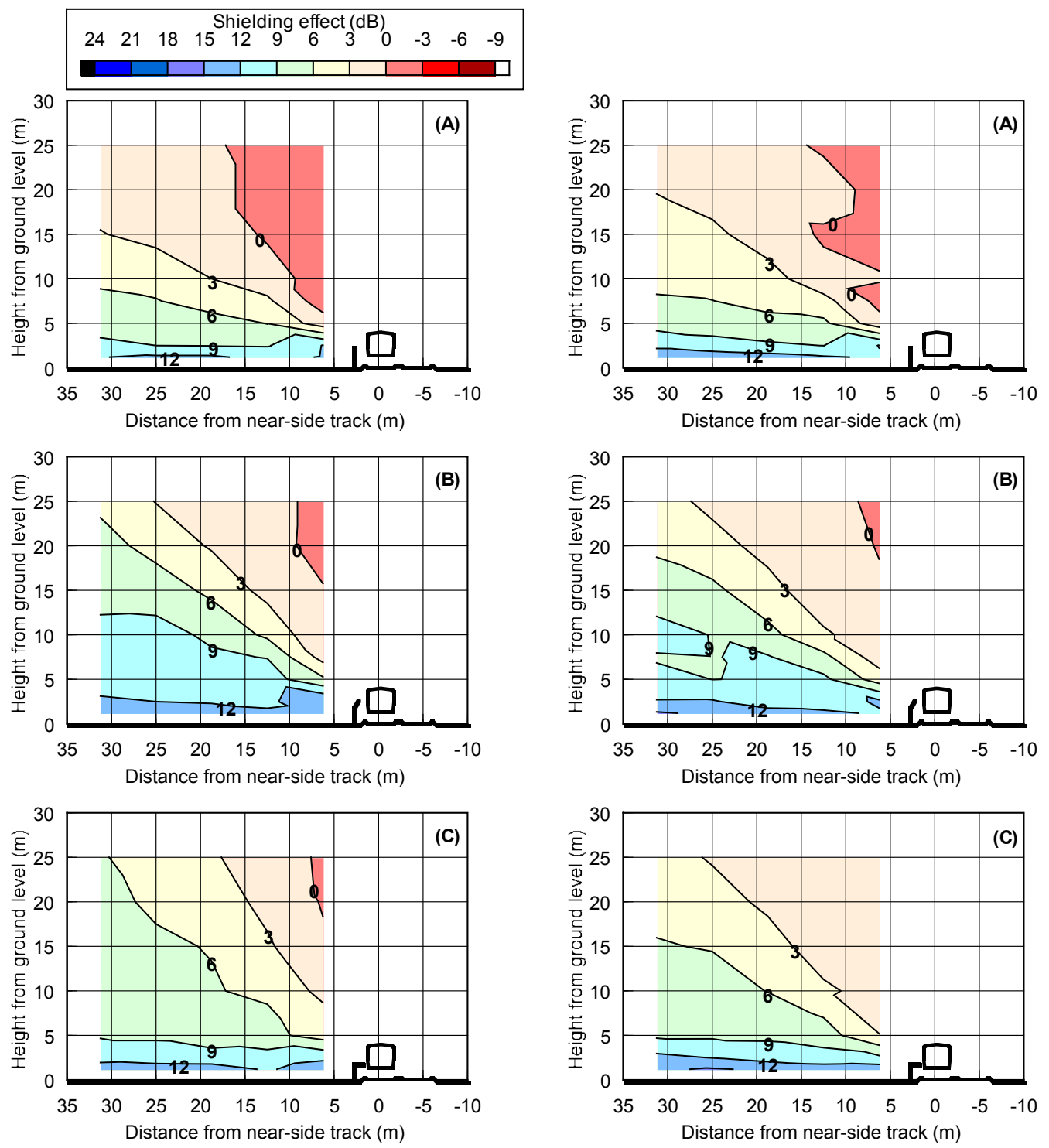

Scale model experiments

2.5D-FDTD analysis

Fig. 5 Spatial distributions of the shielding effects from results calculated by 2.5-FDTD analysis and measured by scale model experiments.

[4] S. Sakamoto, "Phase-error analysis of high-order finite difference time domain scheme and its influence on calculation results of impulse response in closed sound field," Acoust. Sci. \& Tech., 28, 295-309 (2007).

[5] S. Sakamoto, "Calculation of sound propagation in threedimensional field with constant cross section by Duhamel's efficient method using transient solutions obtained by finitedifference time-domain method," Acoust. Sci. \& Tech., 30, 72-
$82(2009)$

[6] P. Jean, J. Defrance and Y. Gabillet, "The Importance of source type on the assessment of noise barrier," J. Sound Vib., 226, 201-216 (1999).

[7] D. Duhamel and P. Sergent, "Sound propagation over noise barriers with absorbing ground," J. Sound Vib., 218, 799-823 (1998). 